\title{
Semantic and Logic Modeling of Disaster Simulation for Multi-agent Systems
}

\author{
Claire Prudhomme, Christophe Cruz, and Frank Boochs
}

\begin{abstract}
Disaster management is a complex collaborative process involving several stakeholders from different domains and requiring preparation for designing action plans. Computer simulation of such collaborative process allows globally assessing the efficiency of such preparation. Studies have shown that multi-agent systems (MAS) are well suited for identifying an optimal strategy or potential issues in the context of one or several action plans. Thus, our approach relies on a MAS for simulating action plans. We consider combining such an approach with Semantic Web technologies, in order to define the conceptual simulation model according to the preparation model of disaster management. The knowledge base is expressed through two different ontologies (semDM to model the preparation results and semMAS to model the simulation) that will be discussed in this paper. On top of these knowledge models, domain-specific constraints allow for checking consistency, and logic rules are used to define the semMAS modeling according to semDM.
\end{abstract}

Index Terms-Disaster management, multi-agent simulation, semantic web technologies, modeling.

\section{INTRODUCTION}

Disaster management comprises four steps: mitigation, preparedness, response, and recovery [1]. This cycle of four steps aims at improving the critical step of response from the analysis of risks and past experiences. Response step requires the most efficiency. However, the constraints of time and the stress during this step limit its efficiency. That is why the key step of the cycle is the preparedness, which allows stakeholders to get ready by organizing, preparing, and training to respond efficiently to a disaster.

The preparation is done both collaboratively through the distribution of responsibilities between the different stakeholders (e.g., communal plan in France [2]) and individually by each stakeholder through plan and protocol design according to their business knowledge (e.g., firefighter protocol [3]). However, during the response, all the stakeholders have to work collaboratively to face the disaster, and actions of one can impact the other. That is why the application of the individual preparation produces some problems or a loss of efficiency.

To gain experience without waiting for a new disaster, drills are organized. Drills are training that aims at assessing

Manuscript received December 15, 2018; revised May 1, 2019. This work was supported by the German Federal Ministry of Education and Research ("Bundesministerium für Bildung und Forschung") [grant numbers 03FH032IX4].

Claire Prudhomme and Frank Boochs are with the institute i3 mainz of the University of Applied Sciences, Lucy-Hillebrand-Str. 2, 55128 Mainz, Germany (e-mail: claire.prudhomme@hs-mainz.de, frank.boochs@hsmainz.de).

Christophe Cruz is with the Laboratoire d'Informatique de Bourgogne (LIB) - EA 7534, University of Bourgogne Franche-Comté, Bâtiment i3M rue Sully, 21000 Dijon, France (e-mail: christophe.cruz@ubfc.fr). the preparedness of the different response stakeholders that sometimes includes the population. However, the high cost of drills limits their quantity and their quality. The disaster management faces lack of collaborative and shared point of view during the preparation that produces a loss of efficiency to respond to a disaster. This lack of shared point of view due to the low number of collaborative drills must be fulfilled by another way. In drills, a shared feedback on experience requires applying collaborative and individual disaster preparedness to assess its result with a shared point of view.

The collaborative and individual preparation makes intervention of a set of organizations (different actors with different roles and responsibilities), a set of plans (distribution of task responsibilities), and a set of protocol (set of actions and resources required to achieve a task). Among the different business domains intervening in the disaster management, some have predefined protocols, and some others use their business knowledge to build protocols according to the disaster situation. A disaster situation is composed of a set of events that impact the population and the infrastructures in the considered geographic area. Studies have shown that multi-agent simulations (MAS) are well suited for identifying an optimal strategy or potential issues in the context of one or several action plans. The level of granularity provided by a multi-agent simulation represents the diversity of organizational structure, actors, and behaviors representing the preparation result. The existing works have a conceptual model designed for a specific use case or proposed metamodel allowing for designing a diversity of use cases; however, these works do not provide approach allowing the generation of a conceptual model from an explicit preparation model. A conceptual model for a realistic simulation of preparation results has two prime input requirements. First, geospatial data containing the location, population, and infrastructure information must be gathered and linked to information related to disaster situation and its impacts. Second, information and knowledge about organizational structure, actors, tasks, actions, and resources from different stakeholders must also be related to the disaster management. According to the location and the type and gravity of the disaster situation, the associated preparation model varies, fitting the requirements of the situation. To simulate preparation result, its conceptual and implemented models have to be adapted according to various possible disaster situations and, thus, various preparation models. The previously cited data and information allow for configuring the preparation model according to the situation. That is why these different elements must be gathered and explicitly linked. Gathering and linking this information imply the following:

- The requirement of disaster management domain modeling 
- The requirement of heterogeneous data integration from different sources

Finally, the simulation has two requirements at the level of its analysis to assess its result and provide a feedback allowing the disaster community to improve their preparation. Although the simulation assessment is out of the scope of this paper, the conceptual simulation model design must allow the following:

- The observation of task failures and their cause to highlight a lack of preparation or a problem of feasibility in the achievement of the preparation

- The global analysis of response efficiency accordingly to the purpose of disaster response, which are the civil and environmental protection, limited victims, loss of life, and damaged infrastructures

Our approach (i) facilitates the design of explicit preparation models through heterogeneous data integration and vocabulary from disaster management; (ii) automatically generates the conceptual simulation model, thanks to a definition done from the preparation model; and (iii) automatically generates the implemented model corresponding to the conceptual model. We consider the Semantic Web technologies to design two semantic metamodels: one called semDM for the explicit preparation models and one called semMAS for defining a conceptual simulation model through logic rules according to semDM. This paper presents, firstly, works related to the disaster preparedness and, secondly, the different steps and components of our approach, which allows the simulation of preparation models.

\section{RELATED WORK}

In the context of preparation assessment, some approaches use static model (as Fault trees of the FunctionInteraction-Structure method [4]) and address the requirements of disaster management domain modeling to assess the feasibility of plans according to an environment state. The limits of the static models reside in their partial view of the situation by taking into account only aspects of actions and interactions on one environment state that does not allow for taking into account the population behavior that impacts the responders and for providing a global efficiency assessment of response that requires analyzing the situation evolution. This evolution required a dynamic aspect provided by simulation techniques. The first section highlights the most adapted simulation technique for disaster response and its limits of model adaptation at a level of a domain rather than at a level of use case. The second section presents limits of approaches, which aims at providing modeling flexibility to allow application in different disaster response cases.

\section{A. Simulation of Disaster Response}

Among the simulation techniques used for the disaster management, the multi-agent simulation is the most used simulation technique during the preparation to assess strategies and plans of disaster response [5]. The granularity of MAS allows representing not only disaster management stakeholders, their organizational structure, their interactions, and their actions that come from their preparation and knowledge according to the situation but also population behavior according to their specificities. Among multi-agent simulation of disaster response, a majority of them aims at determining the optimized solution to a specific problem [6]. The most addressed specific problem is the allocation of resources both in terms of human rescuers [7], [8] and robotic rescuers [9] and of resource means [10], [11]. The simulation efficiency result for the optimization of allocated resources and for planning of response action is assessed in terms of time and success quantity (e.g., ratio of rescued people) according to the purpose of the strategy assessed by the simulation or the use case addressed by the simulation. This type of simulation addresses mainly the rescue strategies. Another set of simulation focuses on evacuation simulation ([12]-[16]) by taking into account some aspects such as the behavior of the population, the traffic, communications, and prepared plans.

The limit of these simulations to provide an experience feedback is the design of the conceptual model and its implementation, which are specialized to a use case or a specific problem and cannot be adapted to the diversity of disaster situation and response strategies.

To solve this lack of flexibility and reusability of simulation models and their implementation in a same domain of application, some approaches presented in the next section have developed metamodel, ontologies, and automatic process to improve the reusability and the extension to different use cases.

\section{B. Approaches to Automate and Facilitate the Multi- Agent Simulation Design}

To solve the problem of reusability and adaptation to diverse contexts, several approaches have used the methodology of developing a formal metamodel as a base to define the conceptual simulation model independently from its implementation model, associated with an automatic process that generates the implementation model. The formalism used to design the metamodel varies between diagram-based modeling language (as UML [17] or its extension like ERE-ML based on MAS-ML and TAO formalisms [18]) and ontologies (Einsim ontology [19], [20]). Ontologies have the benefit of providing a semantic formalism that facilitates the reuse of information content between different systems [19]. These approaches facilitate the development of simulation and the reusability through the metamodel for different use case. However, they do not address the problem of designing the conceptual simulation model that requires a lot of effort to be adapted to the different use cases. According to the geographic location and the specificities of a disaster, actors and their associated plan and business knowledge will change and will require a new simulation model. The approach presented in the next section addresses this problem by (i) facilitating the integration of data and information about disaster management from different sources, (ii) using logic rules and constraints to design a conceptual multi-agent simulation model from integrated information, and (iii) generating the implemented simulation model. 


\section{APPROACH}

This system aims at generating a simulation of the preparation results to face a disaster from the integration of real data (geospatial data, population data, risk data, etc.) and information (task plan, action protocol, business rules, resources, etc.) related to the disaster management. The purpose of such a simulation is to assess the preparedness to provide a support for improving it. The system is composed of a knowledge base containing two main metamodels. The first one is the semDM metamodel that allows for representing models of preparation according to disaster risks. It provides a disaster management vocabulary to integrate and manage related data and information. The second one is the semMAS metamodel that allows for representing conceptual models of multi-agent simulation. A conceptual MAS model aims at designing model according to a preparation model. The achievement of the system goal is done through a sequence of three processes. Firstly, a data integration process facilitates the design of a model preparation. Secondly, a reasoning process dynamically generates a conceptual multi-agent simulation model according to a preparation model and a disaster event. Finally, a process interprets automatically the conceptual multi-agent simulation model to generate the simulation programming. Figure 1 presents the overview of this approach.

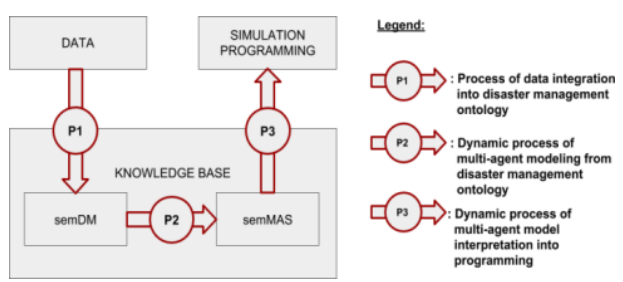

Fig. 1. System overview.

\section{A. Data Integration Process}

The data integration process aims at integrating heterogeneous data related to the disaster management domain into the semDM metamodel. The first section describes the type of information contained in the data concerned by the integration process. The second section presents the types of data processed and describes their processing.

\section{1) Disaster management-related data domain}

The data related to the disaster management are geospatial data, prepared plan data, and stakeholder data. The geospatial data aims at providing location (e.g., building or resource location) and geographic information (e.g., risk areas). This information allows for representing the real world. The prepared plans provide information about a set of tasks (for the highest level of granularity corresponding to strategic plans) or actions (for the lowest level of granularity corresponding to operational plans). These tasks and actions are defined through an application context, required resources to achieve them, and persons or organizations in charge of them. This information allows for determining the role of each stakeholder during a disaster situation and, thus, distributing tasks to the adapted people. The different stakeholders' information is information about the different organizations that intervene in the disaster response. This information corresponds to the description of people who are belonging to an organization, their role, and the resources that they have (types, quantity). Information about people allows for their representation as an agent in the simulation system with the adapted role according to the real world. Resource information is used in the process of decision-making for applying and managing the response plans.

\section{2) Integration process}

The geospatial data integration into an ontology is a problem addressed in the community of Semantic Web (e.g., a semi-automatic approach [21], an automatic approach [22]). Two different approaches have been used to integrate the data: an automatic one and semi-automatic one. The automatic integration process [22] is used for heterogeneous data with a table structure (e.g., shapefiles, database, excel sheet). This approach uses natural language processing and geospatial content (when it is available) to match information from a data with a concept or an individual of an ontology. It then creates an RDF graph with the chosen vocabulary to represent the data content. This automatic approach is useful to process a lot of data with well-named columns (in the sense of complete name). However, some data have column names that are an abbreviation or an incomplete name. In this case, a semi-automatic process is applied to overcome the weakness of the automatic one. The semi-automatic process consists of the definition of a schema mapping between the column name and concepts of the ontology to extract information, transform them into RDF triples, and load them into the ontology.

Thanks to the combination of these two approaches, the heterogeneous data content is integrated into the semDM metamodel presented in the next section.

\section{B. SemDM Metamodel}

The semDM metamodel gathers a representation of information of the geospatial domain and disaster management domain. The main part of its specification is presented in Fig. 2.

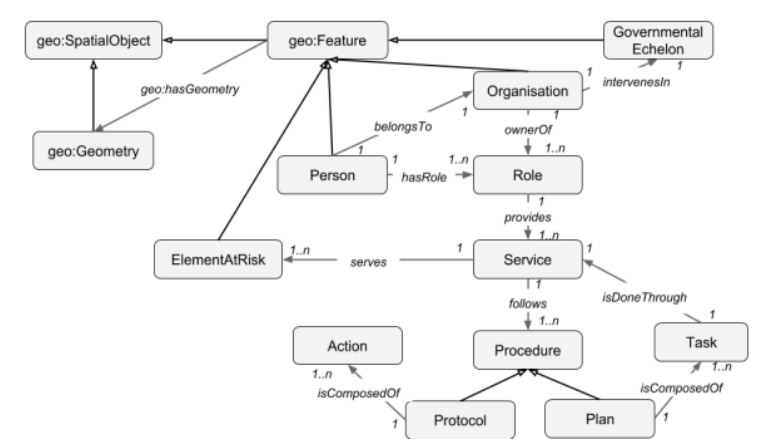

Fig. 2. Specification of the main part of the semDM ontology.

\section{1) Geospatial domain}

Among the different existing geospatial vocabularies (e.g., [23], [24]), the semDM ontology extends the GeoSPARQL vocabulary [25] to represent and manage geospatial information. This vocabulary allows for describing a spatial object through a feature (geo:Feature) and a geometry (geo:Geometry) associated with an object property (geo:hasGeometry). It also provides a set of geospatial 
functions (e.g., contain, overlap, intersect).

\section{2) Disaster management domain}

The semDM ontology is inspired by the metamodel of disaster management presented in [26]. This metamodel provides a domain-specific language for disaster management expertise. The concepts of this metamodel provide a vocabulary to describe the activities of disaster management that facilitates the use of this domain knowledge. The main concepts allowing for representing the response action plans are presented in Fig. 2. The stakeholders of the disaster management are split between organizations and persons. Persons belong to an organization and play one or several roles. Each role provides a set of services that serve elements at risk and follow a procedure. A specification of procedure has been added to the metamodel to distinguish the different types of plans according to their level of granularity. The distinction of procedure types is done through (i) a concept of a plan that is composed of tasks achieved through a service and (ii) a concept of protocol that is composed of actions. The semDM ontology is extended by concepts from the Emergel vocabulary [27] to complete the ontology with a vocabulary specific to the emergency management domain that gathers the main concepts of the response model. The used of "vertical concepts" from Emergel vocabulary aims at specifying some concepts as, for example, (i) Resource from semDM with concepts that inherits from Vehicles and Equipment in Emergel or (ii) Task and Actions from semDM with concepts that inherits from Activities in Emergel.

\section{SemMAS Metamodel}

The semMAS ontology firstly aims at representing a conceptual multi-agent simulation model. Its design is based on the definition provided by Ferber [28] about a multiagent system. This description presents the different components of a MAS. The main component is an environment that corresponds to space and contains a set of artifacts composed of objects and agents. The artifacts are located. Agents are specific artifacts, which are active, whereas objects are passive artifacts. Their distinction is done through a property that assigns operations to only active artifacts, which are the agents. These operations correspond to activities as perceive, produce, consume, transform, and manipulate objects. A concept of relations allows for linking artifacts (and by the way agents) between them. Each agent, each artifact, and the environment have properties that characterize them. The environment also has operators, which represent the application of operations and reactions of the world according to the modification. All of these elements are components of the multi-agent-based simulation model. A simulation model has also some inputs that are initial conditions and parameters.

The semMAS ontology (Fig. 3.) secondly aims at modeling the prepared plans and all other information related to the disaster management to assess the planned collaborative work. For such purpose, the semMAS ontology integrates a representation of the Belief-DesireIntention model [29] corresponding to practical reasoning agent. This model allows for representing (i) the general knowledge of an agent and its knowledge about the situation

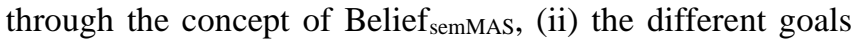
that can be pursued by an agent through the concept of

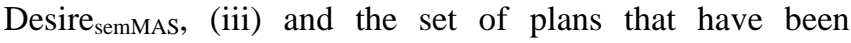

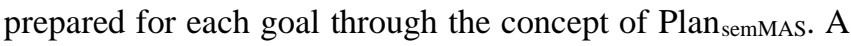
Goal $_{\text {semmAs }}$ corresponds to an active desire and an Intention $_{\text {semMAs }}$ corresponds to an activated plan.

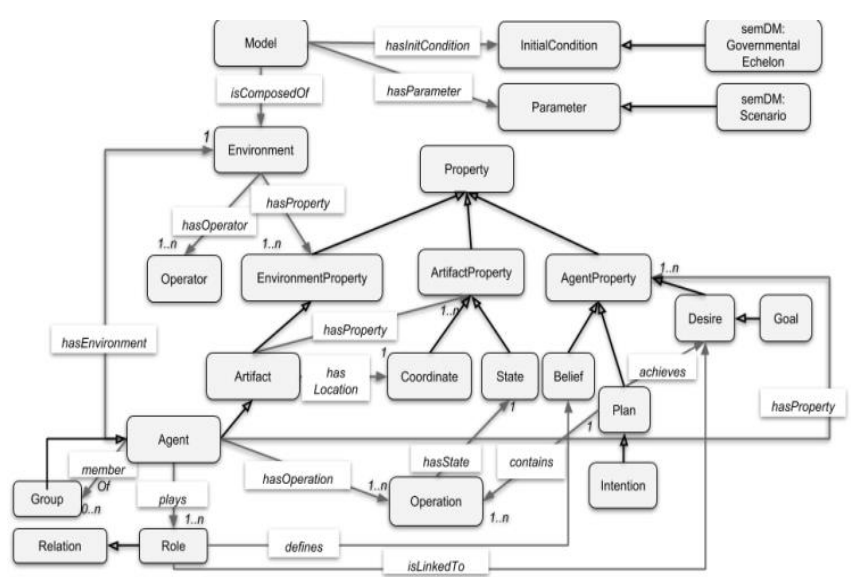

Fig. 3. Specification of the semMAS ontology.

Finally, the semMAS ontology aims at modeling the organizational structure of the disaster management stakeholders. That is why this ontology integrates a concept of group and one of role. A group is defined as a type of agent representing a holonic agent [30].

This semMAS ontology is used to design a simulation model to represent the organizational structure and the response of the disaster management community according to their prepared plan. The model of Belief-Desire-Intention is adapted to represent the prepared plans and the mechanism of their activation according to the knowledge about the situation. The creation of this conceptual multiagent simulation model is done through the process $\mathrm{P} 2$ of dynamic semMAS modeling from the preparation model. This process is presented in the next section.

\section{Automatic Modeling in the SemMAS Ontology}

The automatic modeling process uses preparation model contained in the semDM ontology to design the conceptual multi-agent-based simulation model. The reasoning process applies the logic modeling defined with Shapes Constraint Language (SHACL) [31] on the semMAS ontology from the semDM ontology. This section describes the definition of concepts into the semMAS ontology, which allows the dynamic modeling of simulation components according to the disaster management information. This dynamic modeling is at first a simulation model, then models the environment and its artifacts that compose the model, and finally distinguishes the agents from the other artifacts.

\section{1) Initialization of a model}

The simulation model has two inputs that are initial conditions and parameters. The initial condition for the disaster management simulation corresponds to a governmental echelon. This governmental echelon corresponds to the scale of the simulation. The parameters of the model correspond to a disaster scenario. The scenario corresponds to the timeline of a model. That means a 
simulation model exists for each scenario defined, which takes as an initial condition the smallest governmental echelon corresponding to the scenario.

\section{2) Modeling of the environment with its artifacts}

The definition of model inputs aims at configuring the components of the simulation. The scenario of a model is linked to a disaster. This disaster provides information about the elements at risks that can be impacted by this disaster and the damage that they can undergo in this context. This information allows for defining operators of the environment.

The governmental echelon of a simulation model represents the space of an environment. Thanks to a definition using geospatial functions (as geo:isIn), the reasoner engine can determine the components of the environment from their geographic information. These components are defined as artifacts of the environment and retrieve a discrete location corresponding to the rasterization of their geometry according to the size of the environment given as an input parameter.

\section{3) Modeling of agents}

Among the artifacts of the environment, agents act in the environment. These agents correspond to the stakeholders of the disaster management community. The stakeholders are divided between organizations and persons. The organizations are represented by a group in the multi-agentbased simulation model, and persons are represented by an agent. The link between an organization and a person is kept through the relation of members between an agent and a group.

In the disaster management, a person has a role to provide services. In the multi-agent model, the services are represented by a desire of an agent, a goal that he would want to attempt. An agent plan allowing the achievement of a desire corresponds to the procedures that a service follows. Similar to a service in the semDM ontology, a desire is first linked to a role.

In the second step, shape constraints allowing for directly linking an agent to its desires and its plans are defined. These shape constraints allow the deduction of links between an agent and its attributes (desires and plans) from its links with a role, itself linked to desires and plans.

In the domain of disaster management, a distinction of procedure type is done between plan and protocol that are, respectively, composed of tasks and actions. Actions correspond also to an action that is a subclass of operation in the multi-agent domain.

Disaster management domain tasks are achieved by a service that can be managed by another organization other than the organization that has the plan containing the task. That is why a plan corresponds to a repartition of tasks among diverse organizations. Such a repartition or assignment of a task in a MAS corresponds to the sending of a message containing the service allowing for achieving this task by the organization responsible for this service. The sending of a message corresponds to a communication, which is a kind of agent operation.

Thanks to the definition of another shape constraint, if an agent gets a plan, the operations that compose the action plan become automatically operations of the agent.

This process of modeling produces simulation models composed of an environment, artifacts, and agents. Each of these components is characterized by properties, operations, or operators corresponding to the information about disaster management. To simulate the model, its implementation is created from the semMAS ontology to be then executed and produces an assessment of disaster management response. The process of semMAS interpretation into a programming model is presented in the next section.

\section{E. Automatic SemMAS Interpretation}

The simulation programming aims at producing a simulation execution of the models defined in the semMAS ontology. The simulation execution firstly requires the implementation of a programming model able to represent the conceptual multi-agent-based simulation models contained into the semMAS ontology. It secondly requires a process that interprets a conceptual model into the programming model to initialize the components of the simulation and execute it.

\section{1) Programming model}

The programming model is designed according to a toolbox of implemented behaviors. This toolbox is composed of the basic behaviors that all agents have (e.g., perceiving its environment, decision-making, communicating, or acting). The basic action behaviors as a move and a change on the environment or on an artifact is used to create more complex behaviors and thus provide a set of behaviors corresponding to the set of operations in the semMAS ontology. The implementation of an environment, an artifact, and an agent is done as a pattern that will be filled from the description from the semMAS ontology to allow the creation of a diversity of simulation's components according to their characterization. The pattern of an agent contains an implementation of the Belief-Desire-Intention model.

2) Implementation of model from the semMAS metamodel

The interpretation process uses the implemented patterns to automatically create the simulation program allowing for its execution. This process takes as input the semMAS ontology and creates a program execution for each existing model. The process of interpretation has a bottom-up approach for each model in the sense that it starts from the most specific components to the most general. That means it begins by retrieving all the agents of the model, then initializing their implementation according to their properties and operations, and finally adding all the agents into a list. The second step consists of the same process for each artifact of the model, which is not an agent. The third step is the addition of relation implementation between agents and artifacts. The fourth step is the implementation of the environment according to its operators and its properties that are composed of the list of artifacts and the list of agents. The final step is the execution of the program that aims at providing the assessment of the plans. This assessment is done through the observation of a set of variables representing the result of the response. The main aim of the response is not only to rescue and protect the 
population but also to protect the properties. The observed variables are related to the quantity of population or the surface of properties to protect and the evolution of their status during the evolution of the response (e.g., number of affected people, number of rescued people, the proportion of affected area, etc.). The second output of the simulation allowing assessing plans are the collection of plans that have been triggered but have not been applied and the cause of their failed application.

\section{CONCLUSION}

This paper presents an automated approach based on semantic technologies to automate the simulation of the disaster management plans in diverse scenarios to assess their efficiency. The main contribution of this approach is to allow the direct simulation of disaster management plans from business data and domain knowledge. Thanks to such an approach, people from the disaster management community can use simulations without providing effort of modeling to assess the prepared plans. The main advantage of this approach is its ability to adapt the design of simulation according to different organizational structure and different granularities of plan, thanks to the use of metamodels represented into an ontology. The use of an ontology and a reasoner engine also provides the capabilities to verify the consistency of the model before implementation and execution. This cross-consistency checking on both ontologies avoids risks of a derivation of the system. A second contribution is provided through the ontology of disaster management containing all information of the domain. This domain ontology used in this process can be reused by other systems supporting the disaster management, for example, by a system that supports the decision-making of actions according to a description of a disaster and the plans represented into the ontology. The limit of this approach is its translation process of domain ontology into a multi-agent-based simulation model that is specific to the domain of disaster management. This approach is also focused on a global realism based on the decisions made at the highest level rather than a realism of individual behavior at the operational level. This choice aims at producing large-scale simulation providing a global view of the plans' efficiency. Another limit appears also in the data integration process, which requires carefully choosing the data to not increase the complexity and slow down the process of translation with irrelevant data.

The future work of this research is to validate the agentbased model represented by the mapping between the disaster management ontology and the ontology of multiagent simulation. This process of model validation will be applied on a use case based on a scenario used during a drill to compare decisions made by real people of disaster management community and decisions made by an agent during the simulation execution.

\section{REFERENCES}

[1] D. Coppola, Introduction to International Disaster Management, Elsevier, 2011.
[2] Direction de la sécuritécivile, Ministèrefrançais de 1' intérieur de l' outre-meret des collectivitésterritoriales. n.d. "Organisation de La Réponse de SecuritéCivile."

[3] ÉcoleNationaleSupérieure des OfficiersSapeurspompiers (ENSOSP). "GestionopérationnelleetCommandement". https://enasis.univlyon1.fr/clarolinepdfplayerbundle/pdf/35565

[4] N. A. N. Feng, O. Shikulskaya, T. Esmagambetov, T. J. Song, L. Zhang, and W. X. Zhu, "Assessment methods analysis of models reliability of emergency response in emergency situations," DEStech Transactions on Environment, Energy and Earth Sciences, (icepee), 2017.

[5] D. Mishra, S. Kumar, and E. Hassini, "Current trends in disaster management simulation modelling research," Annals of Operations Research, 1-25. 2018.

[6] G. Hawe, C. Graham, T. W. Duncan, and S. C. Roger, "Agent-based simulation for large-scale emergency response: A survey of usage and implementation," ACM Computing Surveys (CSUR), vol. 45, no. 1, 2012.

[7] P. Praiwattana and E. R. Abdennour, "Survey: Development and analysis of a games-based crisis scenario generation system," International Conference on Technologies for e-Learning and Digital Entertainment, Springer, Cham, 2016.

[8] N. B. B. Saoud, T. B. Mena, J. Dugdale, B. Pavard, and M. B Ahmed "Assessing large scale emergency rescue plans: An agent based approach," The International Journal of Intelligent Control and Systems, vol. 11, no. 4, pp. 260-271, 2006.

[9] F. Blatt, M. Becker, and H. Szczerbicka. "Analysing the costefficiency of the multi-agent flood algorithm in search and rescue scenarios," German Conference on Multiagent System Technologies, Springer, Cham, 2016.

[10] G. I. Hawe, G. Coates, D. T. Wilson, and R. S. Crouch, "Agent-based simulation of emergency response to plan the allocation of resources for a hypothetical two-site major incident," Engineering Applications of Artificial Intelligence, vol. 46, pp. 336-345, 2015.

[11] J. Marecki, N. Schurr, and M. Tambe, "Agent-based simulations for disaster rescue using the defacto coordination system," pp. 281-297, 2006.

[12] K. Christensen and Y. Sasaki. "Agent-based emergency evacuation simulation with individuals with disabilities in the population," Journal of Artificial Societies and Social Simulation, vol.11, no.3, 2008.

[13] E. Quagliarini, G. Bernardini, and L. Spalazzi. "EPES-Earthquake pedestrians' evacuation simulator: A tool for predicting earthquake pedestrians' evacuation in urban outdoor scenarios," International journal of disaster risk reduction, vol.10, pp. 153-177, 2014.

[14] T. Zhou, D. Wang, D. Zong, and X. He. "The Study of the responsibility space regionalization of emergency shelters in the urban center and the evaluation of evacuation road based on microscope computer simulation a case of chongqing three gorges square area." Disaster Advances, vol. 5, no. 4, pp. 230-236, 2012.

[15] E. Mas, S. Koshimura, F. Imamura, A. Suppasri, A. Muhari, and B. Adriano, "Recent advances in agent-based tsunami evacuation simulations: case studies in Indonesia, Thailand, Japan and Peru. "Pure and Applied Geophysics, vol.172, no. 12, pp. 3409-3424, 2015.

[16] M. Nagarajan, D. Shaw, and P. Albores. "Disseminating a warning message to evacuate: A simulation study of the behaviour of neighbours," European Journal of Operational Research, vol. 220, no. 3, pp. 810-819, 2012.

[17] K. Mustapha, H. Mcheick, and S. Mellouli. "Smart cities and resilience plans: a multi-agent based simulation for extreme event rescuing." Smarter as the New Urban Agenda, pp. 149-170, Springer, Cham, 2016.

[18] S. HoseinDoost, T. Adamzadeh, B. Zamani, and A. Fatemi, "A model-driven framework for developing multi-agent systems in emergency response environments." Software \& Systems Modeling, pp.1-28, 2017.

[19] V. Balasubramanian, D. Massaguer, S. Mehrotra, and N Venkatasubramanian. "DrillSim: A simulation framework for emergency response drills," in Proc. International Conference on Intelligence and Security Informatics, pp. 237-248, Springer, Berlin, Heidelberg, 2006.

[20] P. Kruchten, C. Woo, K. Monu, and M. Sotoodeh, "A humancentered conceptual model of disasters affecting critical infrastructures," in Proc. the 4th International Conference on Information Systems for Crisis Response Management (ISCRAM), pp. 327-344, 2007.

[21] C. Debruyne, K. McGlinn, L. McNerney, and D. O’Sullivan, "A lightweight approach to explore, enrich and use data with a geospatial dimension with semantic web technologies," in Proc. the Fourth 
International ACM Workshop on Managing and Mining Enriched Geo-Spatial Data, 2017.

[22] C. Prudhomme, T. Homburg, J. J. Ponciano, F. Boochs, A. Roxin, and C. Cruz, "Automatic integration of spatial data into the semantic web," in Proc. the 13th International Conference on Web Information Systems and Technologies, 2017.

[23] M. Wick, B. Vatant, and B. Christophe, "Geonames ontology," 2015.

[24] S. Auer, J. Lehmann, and S. Hellmann, "Linkedgeodata: Adding A spatial dimension to the web of data," in Proc. the International Semantic Web Conference, pp. 731-746. Springer, 2009.

[25] M. Perry and J. Herring, "OGC geosparql-a geographic query language for rdf data," OGC Implementation Standard, 2012.

[26] S. H. Othman and G Beydoun, "Model-driven disaster management," Information and Management, vol.5 0, no. 5, pp. 218 - 28, 2013.

[27] E. R. Azcona, EMS Core Ontology - V2

[28] J. Ferber and J. F. Perrot. "LesSystèmes multi agents: versune intelligence collective," InterEditions, 1995.

[29] M. Bratman, "Intention, plans, and practical reason," 1987.

[30] N. Gaud, "Systèmes multi-agents holoniques: De L'analyse à L'implantation: Méta-modèle, méthodologie, et simulation multiniveaux." $\mathrm{PhD}$ thesis, Besançon, 2007.

[31] H. Knublauch and A. Ryman, "Shapes constraint language (shacl)," W3C Candidate Recommendation, vol. 11, no. 8, 2017.

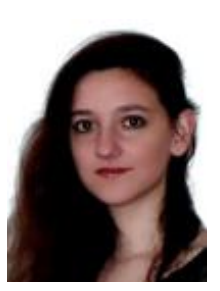

Claire Prudhomme is a Ph.D. student at the University of Burgundy in Dijon (France), and research associate at the i3mainz institute at the University of Applied Sciences in Mainz (Germany). She obtained a master degree with honors in image analysis and artificial intelligence in 2015 at the University of Burgundy. Her research interests include description logics, semantic web, knowledge modeling, simulation, multi-agent systems, and geographic information systems. She works in the semGIS project, which aims at providing a semantic geographic information system with application cases in the domain of disaster management. This research project has led to several publications; the paper entitled "Automatic Integration of Spatial Data into the Semantic Web" at the $13^{\text {th }}$ International Conference on Web Information Systems and Technologies (WEBIST) in April 2017 in Porto (Portugal). This paper has received the best student paper award.

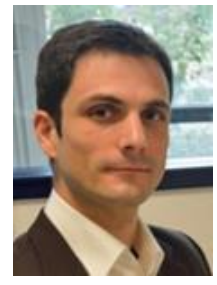

Christophe Cruz is an associate professor at the laboratory Le2i at the University of Bourgogne, and the vice-president in charge of Digital Policy and Information Systems of the University UBFC (Université Bourgogne Franche-Comté). He leads the cluster team "Smart Environment" of the laboratory Le2i. Christophe Cruz obtained a Ph.D. in computer science in 2005 and a habilitation degree in 2012 at the University of Bourgogne. He leads both academic and industrial projects ranging from knowledge modeling to big data processing. his research interests include description logics, semantic web, data science, recommender systems, spatio-temporal data, text processing, knowledge modeling, and user-centric systems.

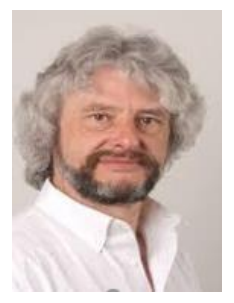

Frank Boochs is a professor and managing director of the i3mainz institute at the University of Applied Sciences in Mainz (Germany). He obtained an engineer diploma in Geodesy in 1978 and a Ph.D. in image processing in 1984 at the RFW University in Bonn (Germany). His research interests include 3D techniques of measure, image analysis, optical technologies, semantic modeling, and spatial humanities.

Prof. Dr.-Ing. Boochs was head of image processing group in the Institute for applied geodesy in Frankfurt, Germany from 1987 to 1993 . He became in 1993 a professor for image processing, photogrammetry, and Geoinformatics in the University of applied sciences in Mainz until 2001. In 1998, he co-founded the Institute for spatial information and surveying technology and since 2001, he is a professor for applied informatics in the same university. Since 2002, he is a managing director of the Institute for spatial information and surveying technology and he has acquired more than 30 projects with a cumulated funding amount of about four million euros. In 2012, he received the science award of the state Rhine-palatinate and the science and literature for outstanding research and teaching activities. 